\title{
Lung CT Parenchyma Segmentation using VGG-16 based SegNet Model
}

\author{
Abdulhadi Omar \\ Faculty of Engineering \\ Sebha University \\ Sebha, Libya
}

\begin{abstract}
Lung parenchyma segmentation is a very important stage in every CAD system for lung cancer detection. In this paper, we propose a new method for CT lung Parenchyma segmentation using the deep SegNet neural network with VGG-16 model. Firstly, 120 CT lung images were collected for the training phase and their ground truth maps were obtained using manual segmentation. Secondly, the training images alongside their corresponding ground truth label images were used as input to the VGG-16 based SegNet model. Finally, 60 CT lung images were collected to validate the performance of the model. The experimental results showed that an accurate segmentation with an average dice similarity index equal to 0.9586 is achieved.
\end{abstract}

\section{Keywords}

Lung CT, Parenchyma, Semantic Segmentation, Deep learning, SegNet, Vgg16.

\section{INTRODUCTION}

Lung cancer is by far the most outspread disease $(11.6 \%$ of the total cancer cases in the world) and the main cause of death [1]. It is attributed to the growth of a malignant tumor in lung tissues. Computerized tomography (CT) imaging is a procedure that utilizes a special $\mathrm{X}$-ray equipment to produce three dimensional set of images from inside the body [2]. A computer aided diagnosis (CAD) system is an essential medical diagnosis tool that plays the role of the physician in deciding the malignancy likelihood of a pulmonary nodule. The importance of developing an effective CAD system lies in the fact that it can raise the patient's opportunity of survival. The performance of any CAD system strongly relies on the segmentation accuracy of the parenchyma part and any mistake involved in this process can negatively affect the performance of the final classification stage. Segmentation of the lung parenchyma is not a straightforward task especially for a parenchyma with a high dense of abnormalities and in case of pulmonary nodules attached to the chest wall, which are missed out by a CAD systems [3, 4]. Moreover, the lung parenchyma needs to be extracted from the bronchus regions that are often confused with the lung tissue [5]. This led to the evolution of a great area of research in automated segmentation of the lung parenchyma. Most of the segmentation methods in the literature are non-trained methods like thresholding based methods $[6,7]$ that relied on the fact that the lung regions have lower intensity values compared with other body regions contained in the CT image. But the limitation of thresholding based methods that appears when the image doesn't have significant grayscale difference or there is overlap between the grayscale pixel values between different objects in the image. Other non-trained methods like region based segmentation such as region growing [8], which is sensitive to background noise and the initial seed point and
K-mean clustering [9] in which it's to predict the number of clusters (K-value and also sensitive the initial seed values and edge detection methods such as watershed technique, which is sensitive to noise and smooth transition in the image were also introduced in [10]. Deep learning is a recent family of machine learning that has proven superiority in many classification and pixel wise segmentation applications. It's superior to other traditional segmentation methods mentioned above as it can detect different objects, determine their shape and the direction of the object. Unlike conventional neural networks like MLP, Deep neural nets deal directly with images as input instead of the manually extracted features since they already have multiple processing layers arranged in three dimensions that extract the high level features within the input image. In [4] a trained deep learning segmentation method was introduced using U-net architecture and a DiceCoefficient index equal to 0.9502 was achieved.

In this paper, we introduce a lung segmentation method using one of the most common deep learning architecture known as SegNet [11] using the layers of a typical deep learning convolutional neural network model named as VGG-16 with an encoder depth equal to 5. The paper is organized in 6 main parts: in the second part, the dataset used in this work is presented. The third part covers the architecture of the SegNet model in details. In the fourth part the training options of the model and the resultant segmentation accuracy using the LIDC image dataset and our VGG-16 based SegNet model. In the fifth section, conclusion of the preset work and future plan are stated.

\section{DATA COLLECTION}

In order to evaluate the performance of the proposed method, 180 CT lung images were collected from the LIDC database [12]. This database is available for public in the National Biomedical Imaging Archive (NBIA). Images in this database are of equal size of $512 \times 512 \times 8$ bit and they are available in Digital Imaging and Communications in Medicine (DICOM) format.

\section{METHODS}

SegNet is a deep convolutional neural network for semantic pixel wise image segmentation. As show in Fig.1, It is made up of a number of layers representing the encoder network and a corresponding decoder network arranged one after another and succeeded by a final pixelwise classification layer [11]. Each encoder performs convolution, batch normalization and a ReLU non-linearity, then performs max pooling to the result, while storing the indices of the values extracted from each feature map. Decoders do the same job as encoders but in reverse order, the difference is that they don't have a non-linearity, and they utilize the indices from the encoding stage to upsample their input [13]. A typical SegNet is made up of 13 convolutional layers from VGG-16 with 
encoder decoder depth of 5 as shown in Fig.1. The network depth is a parameters that controls how many times the downsampling or upsampling process is applied to the input image. The encoder part of the network does the job of downsampling the image by a factor of $2 \mathrm{D}$, where $\mathrm{D}$ refers to the depth of the encoder. The decoder part of the network is responsible for upsamiling the encoder output by the same factor as the encoder. Our lung dataset comprises 180 lung CT images divided into 120 images for training and 60 images for validation. The size of the images was reduced to $250 \times 250$ to speed up the training process. Prior to the training phase, all training images were subjected to manual segmentation in order to provide the ground truth labels in which each pixel is labeled as parenchyma or background. Every pixel belonging to the parenchyma will take the value 255 . On the other hand, pixels in the background will take the value 0 . Both the CT lung images and their corresponding manually segmented ground truth labels will be used as input to train the VGG-16 based SegNet model.
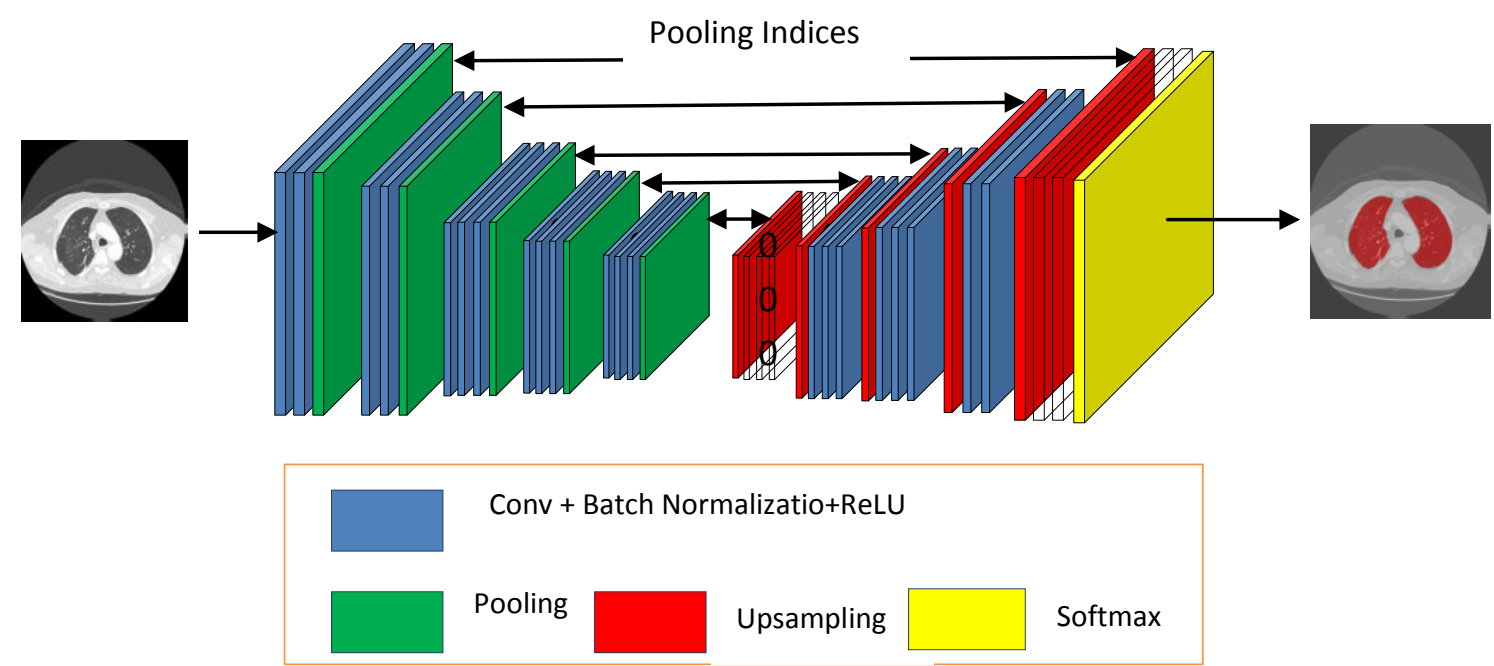

Fig.1 Typical SegNet Architecture

\section{RESULTS AND DISCUSSION}

We have implemented our method using MatlabR2018b. The model is trained on a graphic processor unit NVIDIA GeForce GTX 1060 with 6GB of memory and 1280 CUDA cores. The use of GPU instead of CPU speeds up the training process since GPUs always have much more cores than CPUs and this works faster with applications that involve a huge amount of matrix multiplications and convolutions .The mini batch size was set to 32 , the maximum number of epochs was set to 100 and finally the initial learn rate was set to a fixed value equal to 0.001 . Fig. 2 shows the training progress of our network model. It can be noticed that the training went well with training accuracy up to $99.51 \%$. The detailed progress at every multiples of 10 epoch with training accuracy and the mini batch loss values is demonstrated in Table.1.

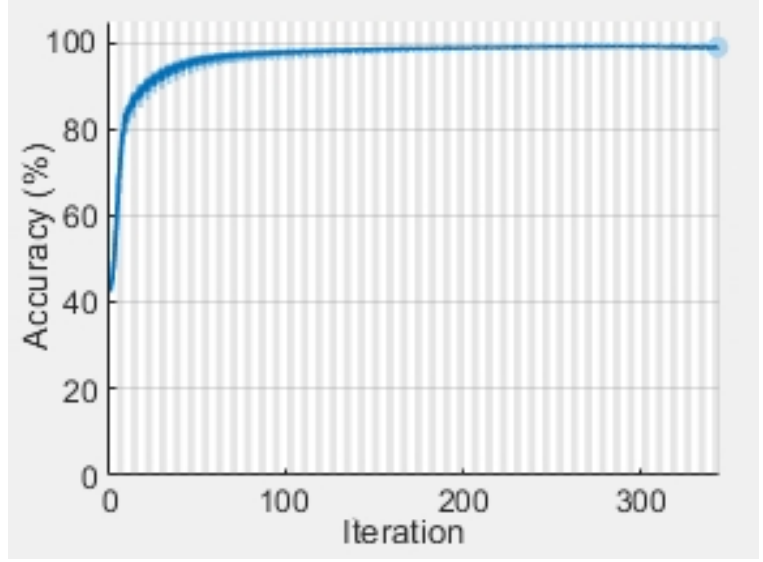

Fig.2 Training progress of the network model

Table.1 Training results of the network model

\begin{tabular}{|c|c|c|c|}
\hline Epoch & $\begin{array}{c}\text { Time Elapsed } \\
\text { (hh:mm:ss) }\end{array}$ & $\begin{array}{c}\text { Mini-batch } \\
\text { Accuracy }\end{array}$ & $\begin{array}{c}\text { Mini-batch } \\
\text { Loss }\end{array}$ \\
\hline 1 & $00: 00: 05$ & $43.60 \%$ & 0.7573 \\
\hline 10 & $00: 09: 22$ & $94.52 \%$ & 0.2852 \\
\hline 20 & $00: 18: 02$ & $96.99 \%$ & 0.1842 \\
\hline 30 & $00: 27: 51$ & $97.85 \%$ & 0.1342 \\
\hline 40 & $00: 36: 14$ & $98.63 \%$ & 0.1025 \\
\hline 50 & $00: 44: 07$ & $98.90 \%$ & 0.0819 \\
\hline 60 & $00: 53: 06$ & $99.08 \%$ & 0.0695 \\
\hline 70 & $01: 02: 19$ & $99.24 \%$ & 0.0569 \\
\hline 80 & $01: 12: 03$ & $99.31 \%$ & 0.0519 \\
\hline 90 & $01: 20: 46$ & $99.40 \%$ & 0.0502 \\
\hline 100 & $01: 29: 38$ & $99.51 \%$ & 0.0491 \\
\hline
\end{tabular}

Fig.3 illustrates the segmentation results for different 4 lung CT images. Images in the first column are the raw CT lung images. The second column shows the segmentation results of the SegNet model where the parenchyma is presented by the red color. The third column demonstrates the binary mask of the parenchyma. Finally the segmented parenchymas are shown in the fourth column. It can be noticed from the figure that the network successfully identified the parenchyma part even in the presence of the tumor attached to the chest wall in the second row. 


\subsection{Segmentation Quality Metric}

To assess the quality of the segmented test images, segmentation performance measures are needed. One of the most common metrics used with semantic segmentation is the Sorenson-Dice similarity [14], which is computed as follows:

$$
\operatorname{Dice}(A, B)=\frac{2|A \cap B|}{|A|+|B|}
$$

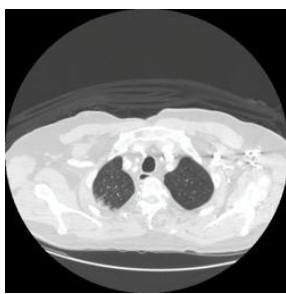

A1

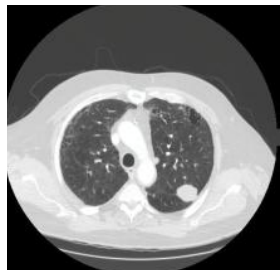

A2

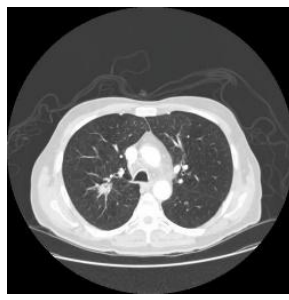

A3

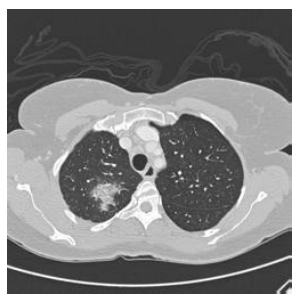

A4

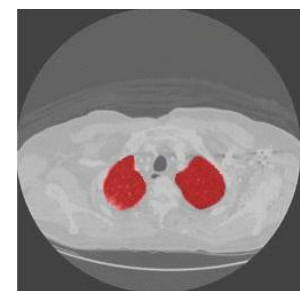

B1

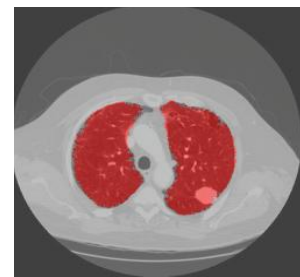

$B 2$

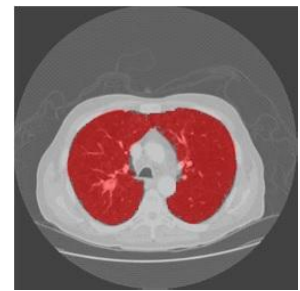

$B 3$

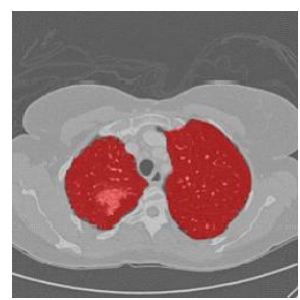

B4

where A denotes the area of the lung parenchyma automatically segmented using our network model, while B denotes the manually segmented lung parenchyma of the same image. The experimental results showed that our SegNet model has successfully segmented the lung parenchyma part with an average dice-coefficient score equal to 0.9586 .

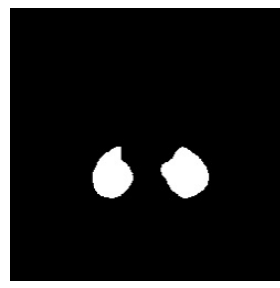

$C 1$

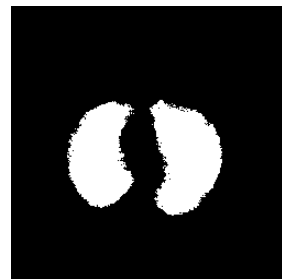

$C 2$

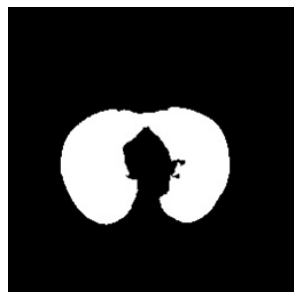

C3

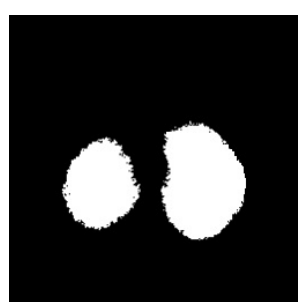

C4

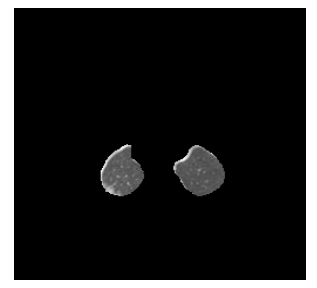

D1

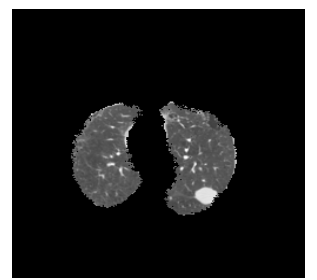

D2

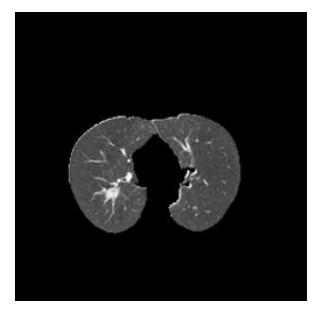

D3

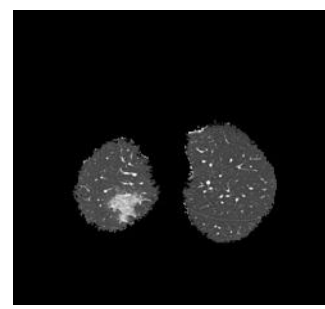

D4

Fig.3 (A1,A2,A3,A4) raw CT lung images, (B1,B2,B3,B4) segmentation using the SegNet model, (C1,C2,C3,C4) binary mask, (D1,D2,D3,D4) segmented parenchyma

\section{CONCLUSION}

In this paper we investigate a lung parenchyma segmentation task using a SegNet model with VGG-16 with encoder decoder depth of 5 . An average dice score coefficient equal to 0.9586 was obtained. The advantage of this method is that it can be applied in a wide range of medical image segmentation tasks. The objective in the next plan is to use this model with 4-D lung images in order and perform segmentation of both parenchyma and tumors and investigate effect of different encoder depths on the segmentation accuracy.

\section{REFERENCES}

[1] Bray F, Ferlay J, Soerjomataram I, Siegel RL, Torre LA, Jemal A, " Global cancer statistics 2018: GLOBOCAN estimates of incidence and mortality worldwide for 36 cancers in 185 countries". CA Cancer J Clin, vol.6, pp.394-424, 2018.

[2] Müller, Pavel; De Chiffre, Leonardo; Hansen, Hans Nørgaard; Cantatore, Angela., "Coordinate Metrology by Traceable Computed Tomography", Technical University of Denmark (DTU), 2013.

[3] P. Hua, Q. Song, M. Sonka, E. A. Hoffman, and J. M. Reinhardt, "Segmentation of pathological and diseased lung tissue in CT images using a graph-search algorithm." Proceedings of the International Symposium on Biomedical Imaging (ISBI '11), pp. 2072-2075, 2011.

[4] Guimei Cui, Liang Wu, Tao Zhou, Yu Gu, Baohua Zhang, Ying Zhao, Dahua Yu, Xiaoqi Lu, and Lixin Gao, 
"Automatic lung nodule detection using multi-scale dot nodule-enhancement filter and weighted support vector machines in chest computed tomography," PLoS ONE, vol. 14, no. 1, 2019

[5] Brahim AIT SKOURT*, Abdelhamid EL HASSANI, Aicha MAJDA, "Lung CT Image Segmentation Using Deep Neural Networks", Procedia Computer Science , vol.127, pp.109-113, 2018.

[6] Lin-Yu Tseng, Li-Chin Huang, "An adaptive thresholding method for automatic lung segmentation in CT images",AFRICON, pp.1-5,2009.

[7] Ying Wei, Guo Shen, and Juan-juan Li, "A Fully Automatic Method for Lung Parenchyma Segmentation and Repairing”, Journal of digital imaging, vol.26, no.3

[8] Juanjuan ZHAO, Guohua JI, Xiaohong HAN, Yan QIANG, Xiaolei LIAO, "An automated pulmonary parenchyma segmentation method based on an improved region growing algorithmin PET-CT imaging", vol.10,pp. 189-200,2016.

[9] Ali Mayya, Mariam Saii, "Lung Detection and Segmentation Using Marker Watershed and Laplacian
Filtering", vol.1, pp.29-42.

[10] Yongqiang Tan, Lawrence H. Schwartz, Binsheng Zhaoa, "Segmentation of lung lesions on CT scans using watershed, active contours, and Markov random field", Medical Physics,vol.40, no.4, 2013.

[11] V. Badrinarayanan, A. Kendall and R. Cipolla, "SegNet: A Deep Convolutional Encoder-Decoder Architecture for Image Segmentation," in IEEE Transactions on Pattern Analysis and Machine Intelligence, vol. 39, no. 12, pp. 2481-2495, 1 Dec. 2017

[12] Lung Image Database Consortium (LIDC): https://imaging.nci.nih.gov/ncia/login.jsf .

[13] X. Zhang, J. Zou, K. He and J. Sun, "Accelerating Very Deep Convolutional Networks for Classification and Detection," in IEEE Transactions on Pattern Analysis and Machine Intelligence, vol. 38, no. 10, pp. 1943-1955, 1 Oct. 2016

[14] Abdel, A. T. \& Allan, H., "Metrics for evaluating 3D medical image segmentation: analysis, selection, and tool. BMC Medical Imaging", Volume 15, pp. 1-29, 2015. 